\title{
Categorizing natural disaster damage assessment using satellite-based geospatial techniques
}

\author{
S. W. Myint ${ }^{1}$, M. Yuan ${ }^{2}$, R. S. Cerveny ${ }^{3}$, and C. Giri ${ }^{4}$ \\ ${ }^{1}$ School of Geographical Sciences, Arizona State University 600 E. Orange St. SCOB Bldg Rm 330, Tempe, \\ AZ 85287-0104, USA \\ ${ }^{2}$ Department of Geography, University of Oklahoma 100 East Boyd St., Norman, OK 73019, USA \\ ${ }^{3}$ School of Geographical Sciences, Arizona State University 600 E. Orange St. SCOB Bldg Rm 330, Tempe, \\ AZ 85287-0104, USA \\ ${ }^{4}$ Science Applications International Corp (SAIC) Contractor USGS Center for Earth Resources Observation and Science \\ (EROS), Sioux Falls, SD, 57198, USA
}

Received: 21 February 2008 - Revised: 17 June 2008 - Accepted: 18 June 2008 - Published: 17 July 2008

\begin{abstract}
Remote sensing of a natural disaster's damage offers an exciting backup and/or alternative to traditional means of on-site damage assessment. Although necessary for complete assessment of damage areas, ground-based damage surveys conducted in the aftermath of natural hazard passage can sometimes be potentially complicated due to on-site difficulties (e.g., interaction with various authorities and emergency services) and hazards (e.g., downed power lines, gas lines, etc.), the need for rapid mobilization (particularly for remote locations), and the increasing cost of rapid physical transportation of manpower and equipment. Satellite image analysis, because of its global ubiquity, its ability for repeated independent analysis, and, as we demonstrate here, its ability to verify on-site damage assessment provides an interesting new perspective and investigative aide to researchers. Using one of the strongest tornado events in US history, the 3 May 1999 Oklahoma City Tornado, as a case example, we digitized the tornado damage path and co-registered the damage path using pre- and post-Landsat Thematic Mapper image data to perform a damage assessment. We employed several geospatial approaches, specifically the Getis index, Geary's $C$, and two lacunarity approaches to categorize damage characteristics according to the original Fujita tornado damage scale (F-scale). Our results indicate strong relationships between spatial indices computed within a local window and tornado F-scale damage categories identified through the ground survey. Consequently, linear regres-
\end{abstract}

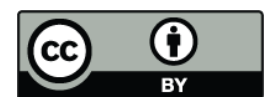

Correspondence to: S. W. Myint (soe.myint@asu.edu) sion models, even incorporating just a single band, appear effective in identifying F-scale damage categories using satellite imagery. This study demonstrates that satellite-based geospatial techniques can effectively add spatial perspectives to natural disaster damages, and in particular for this case study, tornado damages.

\section{Introduction}

A critical problem associated with damage assessment of a natural disaster such as that caused by a tornado is the logistics of quickly coordinating and implementing an extensive ground-based damage survey. In this paper, we introduce a remote sensing approach that can be utilized as a backup or, in some cases such as those involving remote locations, as an alternative to traditional methods of damage assessment. We propose that detailed analysis of remote sensing satellite imagery, because of (a) increasing global ubiquity of satellite imagery, (b) the ability for repeated independent analyses of the data, and, as we demonstrate here, (c) the ability of detailed satellite analysis to verify on-site damage assessment provides an interesting new perspective and aide to researchers.

Ground or aerial damage assessment, such as undertaken after major disasters such as the 3 May 1999 Oklahoma City F5 tornado (Marshall et al., 2005; Spedheger et al., 2002) or the 2004 tsunami (Inoue et al., 2007), is considered crucial to disaster management and planning agencies (as well as to other planning applications) since all hazard mitigation and preparedness programs need to begin with an understanding

Published by Copernicus Publications on behalf of the European Geosciences Union. 
of the risk and its potential impacts through an estimation of the number of people and structures that would be affected by a disaster event. On-site measures of damage have been employed in relation to several severe natural phenomena (e.g., Smith and Robblee, 1994; Reese et al., 2007; Inoue et al., 2007). In the case of a tornado, the Fujita Scale (Fscale), introduced by Fujita $(1971,1973,1981)$, has been the long-time standard damage measurement scale (Doswell and Burgess, 1988) for determining tornado damage based on visual interpretation of wind damage.

The Fujita tornado damage scale categorized tornadorelated damage from F0 to F5 based on the increasing severity of destruction, as primarily evidenced by damage to a "well-constructed" wood-framed house (Fujita, 1971, 1973). There are many challenges in determining the intensity of tornados based on visual interpretation of damage alone. It is understood that the damage caused by severe storms that hit built-up areas is as much a matter of the quality and size/style of construction as it is a function of storm intensity (Doswell, 2003). However, in the case of a tornado, the Fscale has been the standard and accepted measure for evaluating tornado intensity through damage assessment (Bunting and Smith, 1990; Marshall, 2002; Doswell, 2003; Edwards, et. al., 2002). A tornado is normally referred to (and is in this article) by the most intense damage occurring along its track (in this case of the 3 May 1999 Oklahoma City tornado, it is classified as "F5", the greatest amount of damage capable of being assessed).

The enhanced F-scale (or EF-scale) has been recently developed by researchers at Texas Tech University (McDonald and Mehta, 2006) to combine both wind intensity and the types of structures or vegetation being damaged as the traditional Fujita scale. In this study, we selected the use of traditional F-scale over the EF scale because (a) as Doswell and Burgess (1988) stressed, the (traditional) F-scale is a purely damage-related scale, not an intensity or wind speed scale, while the EF scale now takes these factors into account, (b) satellite imagery are available for past tornadic events in which detailed on-site surveys of damage were made (e.g., Marshall, 2002; Speheger et al., 2002), and (c) the traditional F-scale is still being used in many remote parts of the United States and around the world-locations particularly amenable to remote sensing of tornado damage (Bech et al., 2007).

As noted above, a major advantage of the use of remote sensing in the tornado damage assessment is linked to the accessibility to damaged areas immediately after a disaster event. Because of the potential for on-site hazards (e.g., downed power lines, gas lines, etc.), the need for rapid mobilization (particularly for remote locations), and the increasing cost of physical transportation of manpower and equipment, the remote sensing of tornado damage offers an exciting backup and/or alternative to traditional means of damage assessment. Although obviously not a panacea - we fully believe in and accept the need for physical on-site assessmentremote sensing, because of its global ubiquity, its relative low costs and its ability for repeated independent analysis, provides an interesting new perspective and aid to researchers involved with damage assessments. However, caveats do remain: satellite imagery are not yet available for all locations at all times (because of coverage limitations) and, occasionally, costs or acquisition rights for imagery can be prohibitive depending on company, agency and/or government associated with those imagery.

The usefulness of using such imagery in analysis of tornadoes is evident from studies that have shown that, in some cases, damage caused by a given severe storm event can be identified in satellite imagery long after the event. For example, tornado tracks from tornadoes that occurred in eastern Paraguay as long ago as 1965 were still visible in Landsat imagery from the 1970s and 1980s (Dyer, 1988). The spatial mapping of tornado damage is important for a number of interested groups, including insurance companies, farmers, city, and state officials and, particularly, meteorologists and climatologists examining storm morphology and downburst severity (Bentley et al., 2002).

Satellite analysis and interpretation of several types of natural disaster events have begun. For example, a recent special issue of the IEEE Transactions on Geoscience and Remote Sensing contained research on the use of image analysis of earthquake damage (e.g., Arciniega et al., 2007; Sertel et al., 2007; Chaabane et al., 2007), typhoon and hurricane damage (e.g., Barnes et al., 2007) and tsunami damage (e.g, Bovolo and Bruzzone, 2007) among other types of natural disasters. Indeed, the state of art in using remote sensing technologies in damage assessment of many natural disasters has made great strides in recent years, particularly internationally (e.g., Oosterom et al., 2005; Zlatanova and Li, 2008). Yet, perhaps surprisingly, relatively little recent work has been undertaken on tornado damage assessment using satellite technology. Part of that lack of research is that, tornadoes, specifically massive tornadoes such as the 3 May 1999 tornado of Oklahoma City, are limited in their occurrence around the world generally to the United States (Cerveny et al., 2007) although destructive tornadoes have occurred, but rarely, in Bangladesh, China, Great Britain and in western Europe. This recent lack of study of tornado damage assessment by remote sensing technology has occurred despite a long history of using remote sensing imagery in the study of such events. 
In late 1960s, the University of Chicago initiated some aerial survey to examine airflows of tornadoes at multiple scales and their impacts on the ground (Fujita and Smith, 1993). They examined voluminous sets of aerial photographs to correlate wind direction and wind speed to the patterns of damages (structural or natural, e.g., trees) in areas affected by the tornado. Yet, even nearly a half century after such analyses, only a few attempts have applied satellite technology to tornado assessment.

Yuan et al. (2002) demonstrated how tornado damaged areas could be identified in satellite images. In a similar fashion, Jedlovec et al. (2006) used high-resolution imagery from the EOS satellites to study the damage tracks of tornadoes in Missouri and Maryland. However, neither of these papers attempted explicitly to classify damage intensity in images. Moreover, the above studies do not deal with tornado classification categories as evidenced by advanced spatial techniques or statistical models.

Hence, we attempted to develop a remote sensing approach that can be expected to be more straightforward, explicit, and bias free or at least less biased than conducting a damage survey. Remote sensing technology has been used extensively in various disaster applications as a more cost and labor effective and time efficient alternative. In the case of tornado investigation, such analyses can be extremely useful in the detection of tornado paths. For this case study, we selected one of the strongest (in terms of recorded wind speeds) tornadoes ever to strike the United States, the 3 May 1999 Oklahoma City tornado (Cerveny et al., 2006). This tornado was rated F5 on the Fujita intensity damage scale and formed over Grady County near Amber and tracked northeast for thirty-seven miles eventually moving into the Oklahoma City metropolitan area. Bridge Creek, Oklahoma City, Moore, Del City, and Midwest City suffered tremendous damages. Thirty-six direct fatalities and 583 direct injuries were recorded.

We employed ground-surveyed high-resolution data using the Fujita tornado scale after the 3 May 1999 F5 tornado (Marshall et al., 2005; Spedheger et al., 2002) and examined the representativeness of different geospatial indices using a remote sensing change detection approach and GIS technologies. Specifically we categorized tornado intensity values in medium resolution imagery according to the Fujita scale and assessed damages in urban and non-urban areas. We anticipated that degree of damages, debris, and damaged area extents (complex arrangements of damaged structures, trees, and shrubs) in residential areas characterized by geospatial approaches using different window sizes could be correlated to tornado intensity values identified by surveyors on the ground. We employed several geospatial approaches that are capable of describing level of heterogeneity and homogeneity of spatial objects and features that can be linked to chaotic arrangements of structural and vegetated area damages and debris due to a tornado event. The spatial arrangements of damaged areas compared to organized arrangements of other

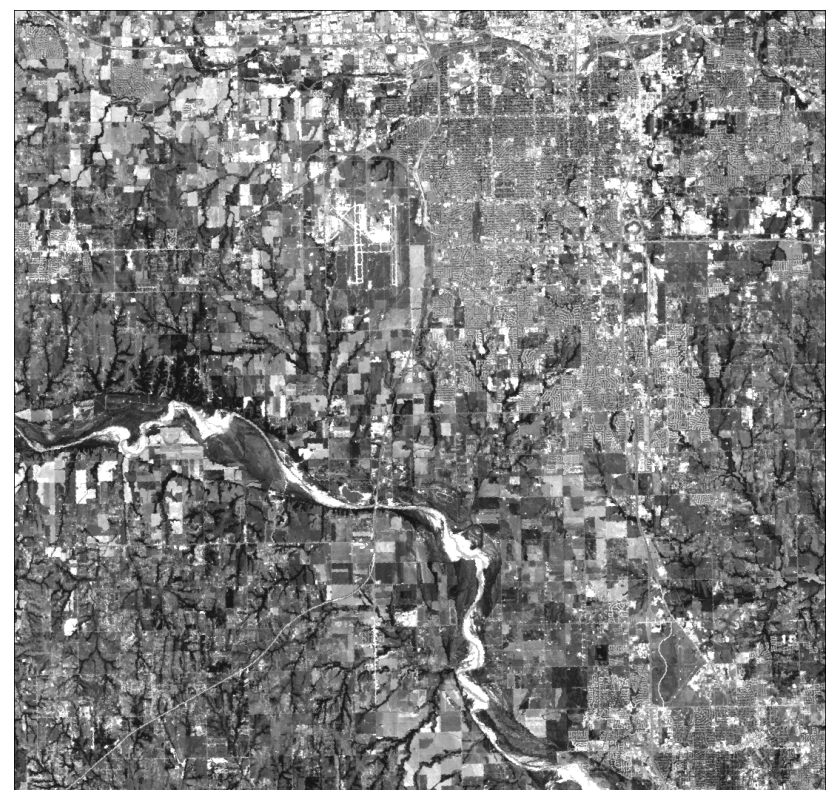

Fig. 1. A gray-scale image of the study area (26 June 1998) displaying channel $3(0.63-0.69)$.

residential, commercial, and industrial areas that are not affected by the same tornado could be expected to be significantly different.

\section{Data preparation and study area}

Landsat Enhanced Thematic Mapper (Landsat ETM+) image data (path 28 and row 35 ) at $28.5 \mathrm{~m}$ spatial resolution with seven channels ranging from blue to thermal infrared portion of the spectrum was used to perform a damage assessment. The thermal channel was excluded in the study due to its coarser resolution. The image data was acquired over central Oklahoma area under cloud-free conditions prior to the 3 May tornado event on 26 June 1998 (Fig. 1) and after the 3 May tornado event on 12 May, 1999 (Fig. 2). The original image was subset to extract the 3 May 1999 tornado damage path (upper left longitude $94^{\circ} 44^{\prime} 47.76^{\prime \prime}$ and latitude $35^{\circ} 28^{\prime} 24.82^{\prime \prime}$, lower right longitude $97^{\circ} 24^{\prime}$ $49.97^{\prime \prime}$ and latitude $\left.35^{\circ} 12^{\prime} 14.98^{\prime \prime}\right)$. The study area covers about $9.4005 \times 10^{8} \mathrm{sq} \mathrm{m}(1097$ columns and 1055 rows $)$. Both images were orthorectified and georeferenced in Universal Transverse Mercator (UTM) projection with a Clarke 1866 spheroid, NAD27 datum, and zone 14 with root mean square (RMS) errors less than 0.5. However, any level of locational errors (RMS errors) in change detection images will produce considerable errors in consequent analysis. It should be noted that error-free geometric correction or $100 \%$ rectification accuracy of any data is practically impossible (Myint and Wang, 2006). Hence, we co-registered both images to 


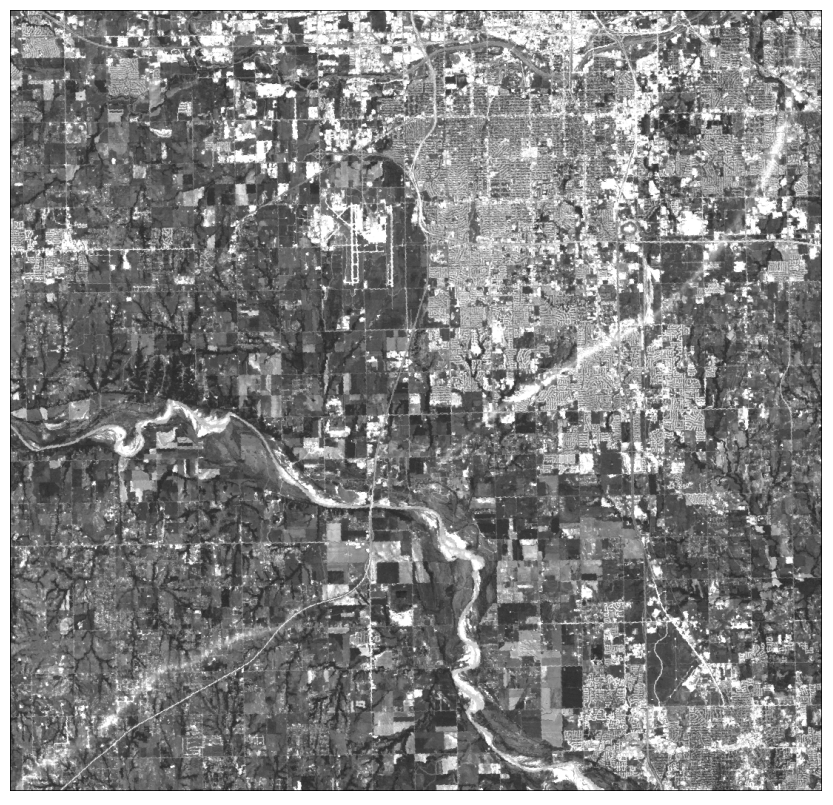

Fig. 2. A gray-scale image of the study area (12 May 1999) displaying channel $3(0.63-0.69 \mu \mathrm{m})$.

minimize locational errors even though both images were orthorectified.

We digitized tornado damage path and co-registered the damage path image with the image acquired after the 3 May, 1999 destructive tornado (Fig. 4). We assigned tornado intensity values using the tornado classification F-scale values identified along the destruction path in the maps and diagrams for the 3 May, 1999 tornado event prepared by the National Oceanic and Atmospheric Administration (NOAA) - National Weather Service (http://www.srh.noaa.gov/oun/ storms/19990503/index.html, Fig. 3).

We then converted the Landsat ETM+ data to apparent surface reflectance using an atmospheric correction method known as the Cos(t) model (Chavez, 1996). This model incorporates all of the elements of the dark object subtraction model (for haze removal) and a procedure for estimating the effects of absorption by atmospheric gases and Rayleigh scattering. Even though data import, image layer stacking, qualitative analysis, and image subset were performed in ERDAS Imagine, conversion from DN values to reflectance was performed one band at a time using ATMOSC module in IDRISI software package. The reflectance data were imported back to ERDAS Imagine for layer stacking. The layer stacked image data was multiplied by 10000 and kept as 16 bit integer data for easy computation and comparison.

\section{Methodology}

We employed a set of geospatial approaches with different window sizes to determine their relations with tornado intensities identified on the ground manually using image differences of Landsat TM reflectance data acquired on 26 June, 1998 and 12 May, 1999. Specifically, we applied the Getis index (Getis and Ord, 1992; Ord and Getis, 1995), Geary's $C$ (Geary, 1954), and two lacunarity approaches introduced in Myint and Lam (2005) and Myint et al. (2006) to categorize the imagery to F-scale damage values. These techniques are discussed in detail below.

\subsection{Change detection}

Digital change detection methods were broadly divided into either pre-classification spectral change detection or postclassification change detection methods (Nelson, 1983; Pilon et al., 1988). Regarding post-classification change detection, two images acquired on different dates are separately classified, and the changes are identified through the direct comparison of the classified information (Howarth and Wickware, 1981).

Spectral change detection or pre-classification techniques rely on the principle that land cover changes result in persistent changes in the spectral signatures of the affected land surfaces. These techniques involve the transformation of two original images to a new single-band or multi-band image in which the areas of spectral change are highlighted (Yuan et al., 1998). We employed the band-subtraction change detection technique using the before and after image data. As described in data preparation we converted brightness values of both images to reflectance data before difference images were generated. The subtracted reflectance data were later stretched to unsigned 8 bit data (Fig. 5). We first exported all difference images to generic binary images since all the algorithms employed to generate spatial-transformed images work with generic binary images.

\subsection{Geospatial techniques}

We employed the Getis index (Getis and Ord, 1992; Ord and Getis, 1995), Geary's C (Geary, 1954), and two lacunarity approaches introduced in Myint and Lam (2005) and Myint et al. (2006) to categorize the imagery to F-scale damage values. The selected geospatial approaches will be hereafter referred to as $G i, G y, L 1$, and $L 2$. We used the Getis index to determine an optimal local window size. Hence, we generated $G i$-transformed images of all bands after image differencing ( 6 difference bands) using different window sizes (i.e., $5 \times 5,9 \times 9,13 \times 13,17 \times 17,21 \times 21,25 \times 25$, $29 \times 29,33 \times 33,37 \times 37)$. The $G i$-transformed images were imported back to Imagine format and re-projected for the regression and correlation analysis. This was because generic binary images do not keep georeferenced information. We 


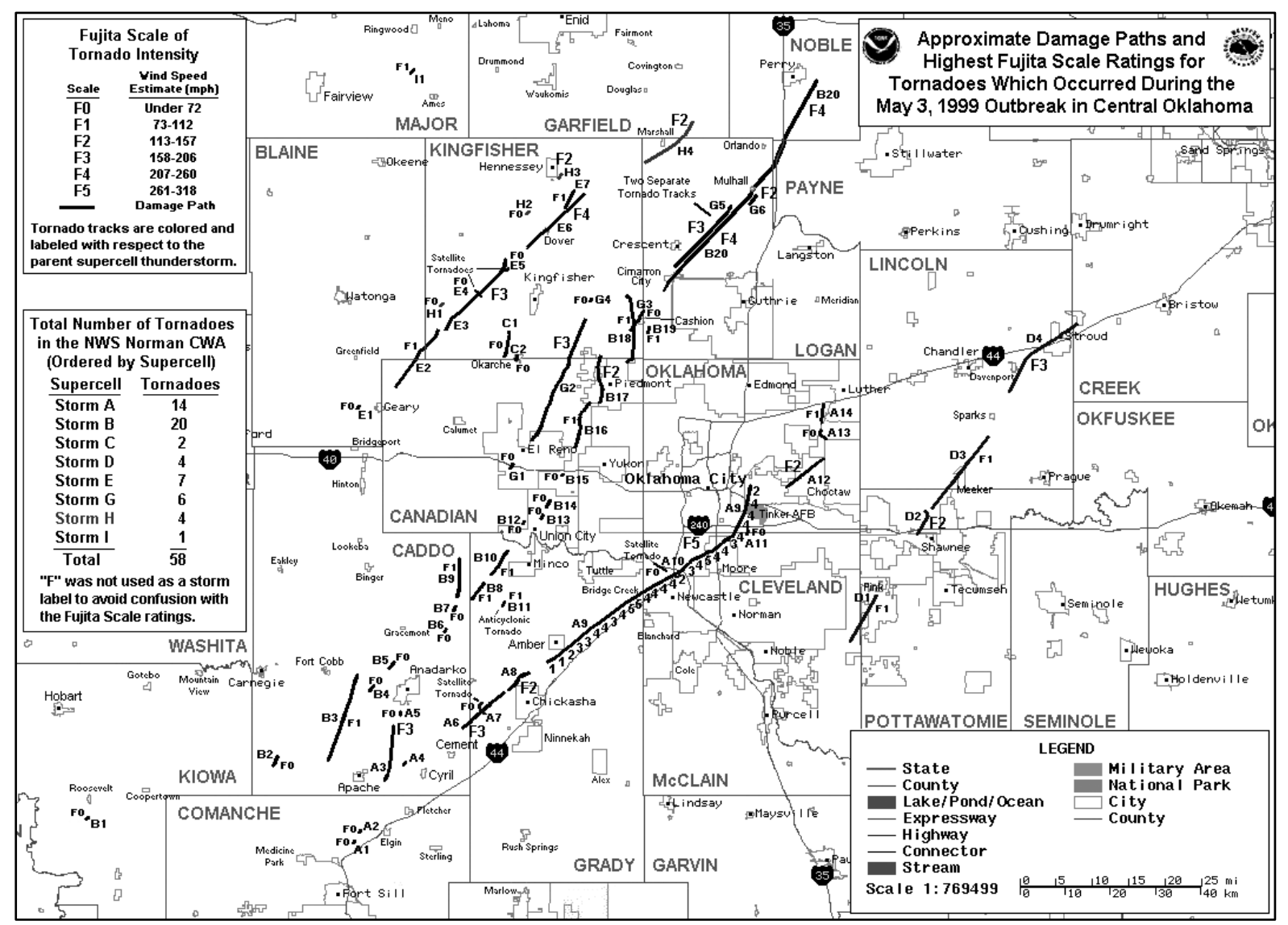

Fig. 3. Tornado outbreak prepared by the National Oceanic and Atmospheric Administration (NOAA) - National Weather Service.

later extracted geospatial indices that intersect with tornado damage classification values identified by the National Oceanic and Atmospheric Administration (NOAA) - National Weather Service. The regression and correlation analysis between Getis indices generated by different windows and tornado F-scale values were performed to determine an optimal window.

After we obtained the optimal window size that has the strongest relation with the digitized tornado intensity values, we generated other geospatial transformed images (i.e., $G y$, $L 1$, and $L 2$ ) using the optimal window size for comparison purpose. Following the same procedure as we did for the $G i$-transformed bands, all other spatial-transformed images were also imported back to Imagine format and re-projected for the regression and correlation analysis. We not only used individual geospatial bands but also used different combinations of geospatial approaches and different bands to obtain potentially effective regression models to predict tornado damage categories.

\subsubsection{Spatial autocorrelation (Geary's $C$ )}

Geary's $C$ is calculated from the following:

$C(d)=\frac{\sum_{i}^{n} \sum_{j}^{n} w_{i j}(d)\left(z_{i}-z_{j}\right)^{2}}{2 \sum_{i}^{n} \sum_{j}^{n} w_{i j}(d) s^{2}}$

where $w_{i j}$ is the weight at distance $d$ so that $w_{i j}(d)=1$ if point $j$ is within distance $d$ from point $i$; otherwise, $w_{i j}(d)=0 ; s^{2}$ is the variance of $z$ values and can be computed as:

$s^{2}=\sum_{i}\left(z_{i}-\bar{z}\right)^{2} /(n-1)$

(see Myint et al., (2007) for detailed computations and worked example) 


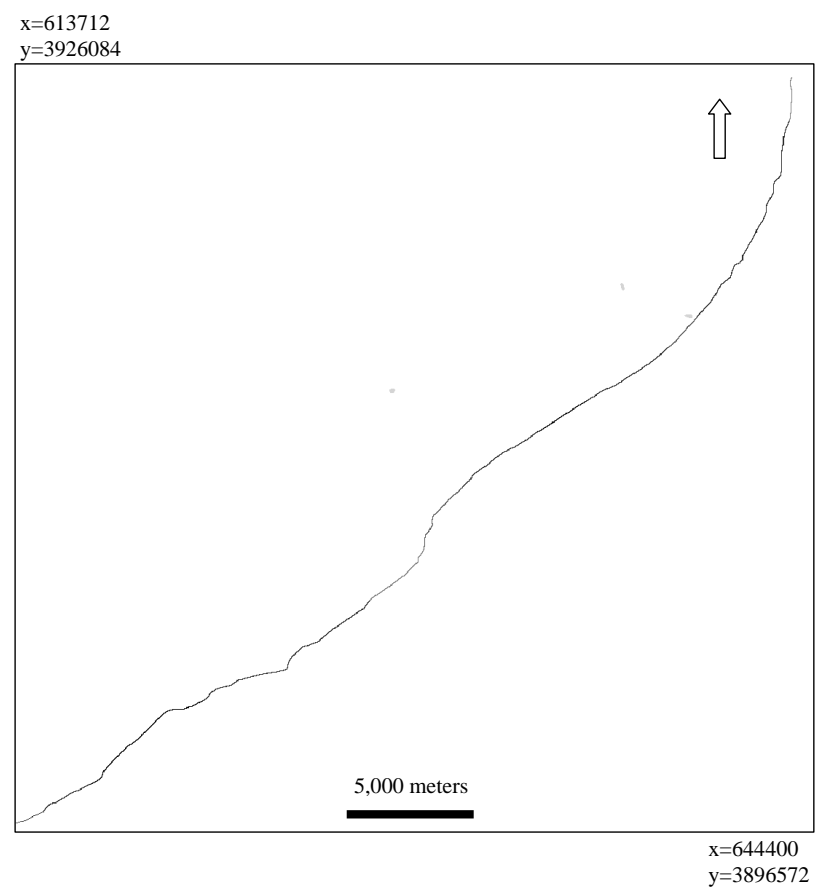

Fig. 4. Digitized 3 May 1999 tornado outbreak and manually assigned intensity values along the path.

\subsubsection{Getis index}

The Getis statistic (Getis and Ord, 1992; Ord and Getis, 1995) is computed as:

$G i(d)=\frac{\sum_{i}^{n} \sum_{j}^{n} w_{i j}(d) z_{i} z_{j}}{\sum_{i}^{n} \sum_{j}^{n} z_{i} z_{j}}, \quad$ for $i \neq j$

The $G i$ is defined by a distance, $d$, within which areal units can be regarded as neighbors of $i$. The weight $\mathrm{w}_{i j}(d)$, is 1 if areal unit $j$ is within $d$ and is 0 otherwise. The relationship among the neighboring points is determined by a distance threshold, $d$. The value of $d$ needs to be defined before computing the Gi. Myint et al. (2007) demonstrated that the shortest distance threshold characterizes the spatial arrangement of objects and features effectively and achieved the highest overall accuracy. Moreover, the overall accuracy consistently decreased with the increasing distance threshold. Hence, we employed the shortest distance (1 pixel or $28.5 \mathrm{~m}$ ) in this study.

(see Myint et al., (2007) for detailed computations and worked example)

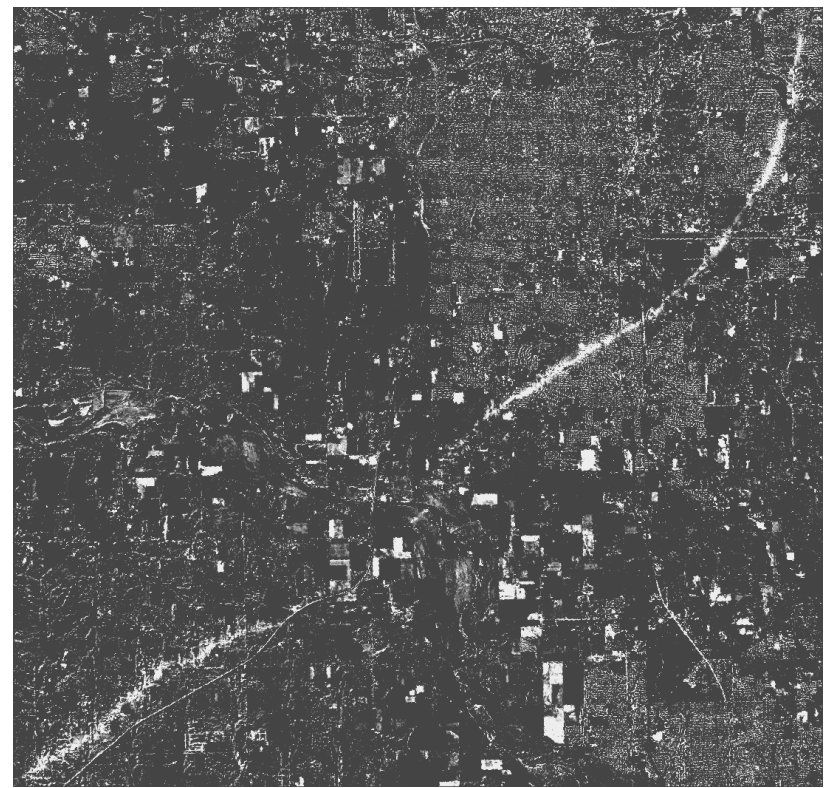

Fig. 5. Image differences of Landsat TM bands acquired on 26 June 1998 and 12 May 1999 displaying channel $3(0.63-0.69 \mu \mathrm{m})$.

\subsubsection{Lacunarity probability approach $(L 1)$}

Voss (1985) proposed a probability approach to estimate the fractal dimension and lacunarity of image intensity surface. The spatial arrangement of the points determines $P(m, L)$. $P(m, L)$ is the probability that there are $m$ intensity points within a box size of $L$ centered about an arbitrary point in an image. Intensity points are referred to as the number points filled in a cube box. Hence, we have

$\sum_{m=1}^{N} P(m, L)=1$

where $N$ is the number of possible points in the box of $L$. Suppose that the total number of points in the image is $M$. If one overlays the image with boxes of side $L$, then the number of boxes with $m$ points inside the box is $(M / m) P(m, L)$. Hence

$M(L)=\sum_{m=1}^{N} m P(m, L)$

and

$M^{2}(L)=\sum_{m=1}^{N} m^{2} P(m, L)$

Lacunarity can be computed from the same probability distribution $P(m, L)$. Hence, lacunarity $\Lambda(L)$ is defined as

$\Lambda(L)=\frac{M^{2}(L)-(M(L))^{2}}{(M(L))^{2}}$

(see Myint and Lam (2005) for detailed computations and worked example) 


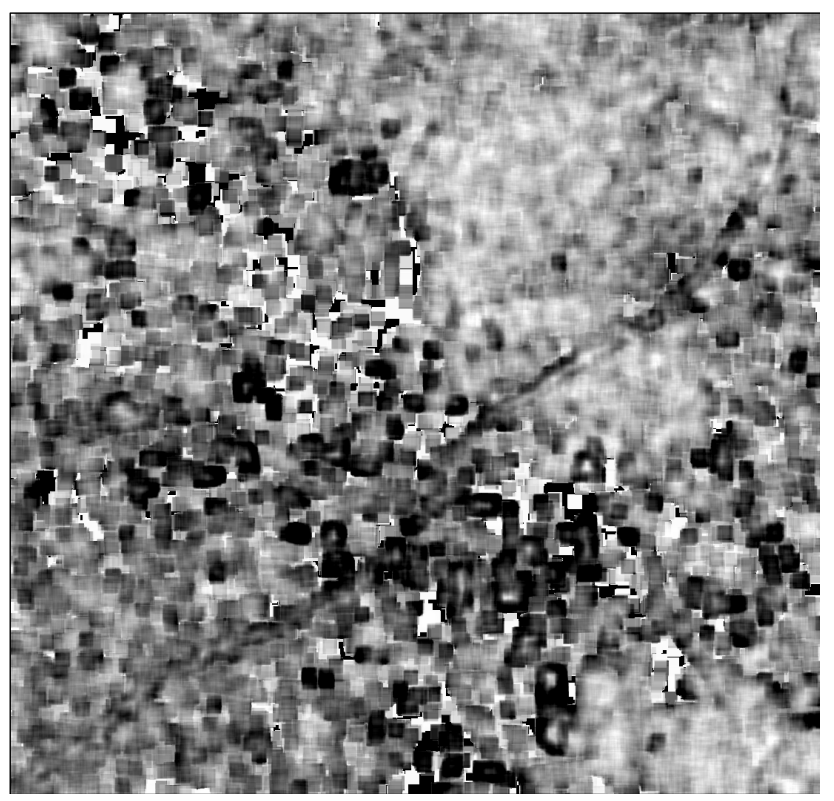

Fig. 6. Geary's $C$ indices of image difference band 3 using $21 \times 21$ window.

\subsubsection{Lacunarity differential box counting approach (L2)}

According to the gliding box algorithm proposed by Allain and Cloitre (1991), $n(M, r)$ can be defined as the number of gliding boxes with radius $r$ and mass $M$. The probability function $Q(M, r)$ is obtained by dividing $n(M, r)$ by the total number of boxes, so that lacunarity at scale $r$ is defined as,

$$
\Lambda(r)=\frac{\sum_{M} M^{2} Q(M, r)}{\left[\sum_{M} M Q(M, r)\right]^{2}} .
$$

A cubic of size $r \times r \times r(r=2,3,4 \ldots)$ is placed over the upper left corner of an image window of size $W \times W$. For each $r \times r$ gliding box, the minimum and maximum pixel values in the gliding box are allowed to fall in box number $u$ and $v$ respectively. Then the relative height of the column is,

$n_{r}(i, j)=v-u-1$

where $i$ and $j$ are image coordinates. Although this calculation gives an accurate height of the column, if the minimum and maximum pixel values fall in the same box, the column becomes negative one; not a problem if using a positive one. We believe this is adequate since the computed value represents the relative height, so when the $r \times r$ gliding box moves throughout the $W \times W$ image window, the following is possible,

$M_{r}=\sum_{i, j} n_{r}(i, j)$

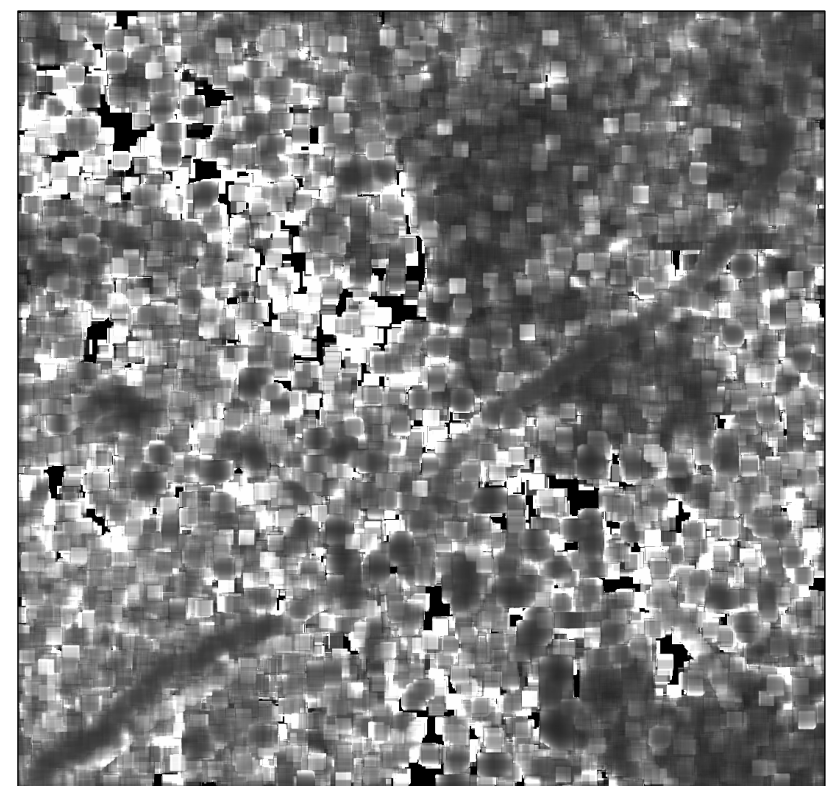

Fig. 7. Getis indices of image difference band 3 using $21 \times 21$ window.

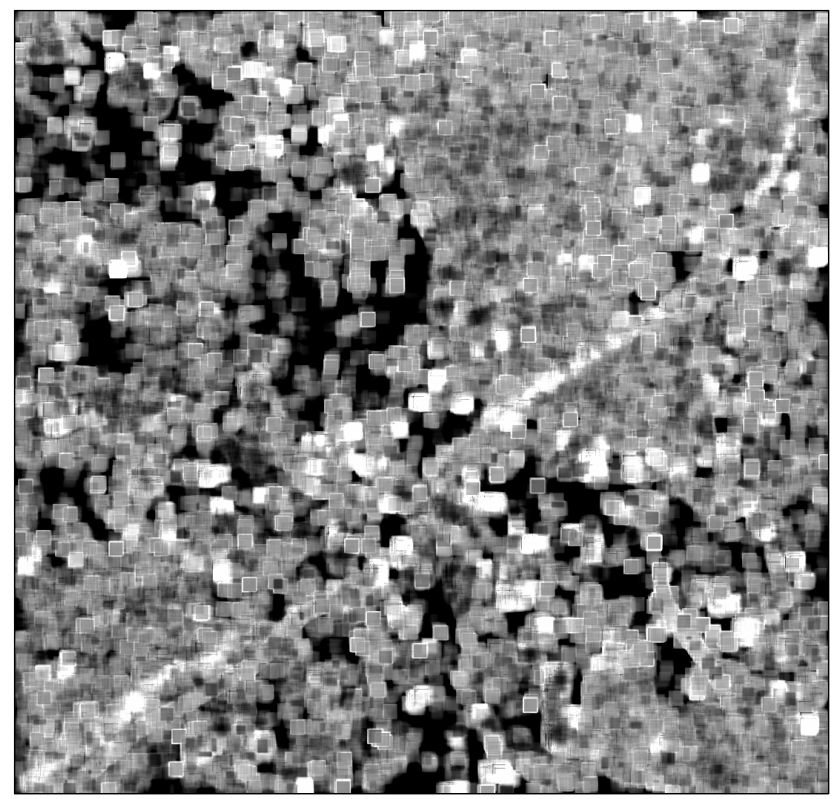

Fig. 8. Lacunarity probability indices of image difference band 3 using $21 \times 21$ window.

The mass $M$ in equation (1) is then replaced by $M_{r}$ to obtain lacunarity $\Lambda(r)$ in the $W \times W$ window (Dong, 2000).

(see Myint et al., 2006 for detailed computations and worked example)

The algorithm assigns the computed geospatial value to the center of the window as it moves throughout the image. Since we lose (n-1)/2 pixels on the top, bottom, left, and right 
Table 1. Summary statistics for the regression models using tornado intensity values and Getis values of band 3 image difference band using different window sizes (i.e., $5 \times 5,9 \times 9,13 \times 13,17 \times 17,21 \times 21,25 \times 25,29 \times 29,33 \times 33,37 \times 37$ ).

\begin{tabular}{llllllllll}
\hline Parameters & \multicolumn{1}{c}{ Window Size } \\
\hline & $5 \times 5$ & $9 \times 9$ & $13 \times 13$ & $17 \times 17$ & $21 \times 21$ & $25 \times 25$ & $29 \times 29$ & $33 \times 33$ & $37 \times 37$ \\
\hline Correlation & -0.09 & -0.09 & -0.40 & -0.49 & -0.53 & -0.51 & -0.45 & -0.4 & $2-0.40$ \\
Coef. of Det. & $0.83 \%$ & $0.89 \%$ & $16.10 \%$ & $24.06 \%$ & $27.78 \%$ & $25.84 \%$ & $20.26 \%$ & $17.71 \%$ & $16.06 \%$ \\
S.E. Estimate & 1.17 & 1.16 & 1.07 & 1.02 & 0.99 & 1.01 & 1.04 & 1.06 & 1.07 \\
Std. of X & 1914.74 & 1276.65 & 986.52 & 814.57 & 616.71 & 503.17 & 408.32 & 380.30 & 360.07 \\
Std. of Y & 1.17 & 1.17 & 1.17 & 1.17 & 1.17 & 1.17 & 1.17 & 1.17 & 1.17 \\
Intercept & 3.60 & 4.95 & 5.88 & 6.18 & 6.53 & 6.62 & 6.59 & 6.46 & 6.36 \\
Coefficient & 0.000246 & 0.000087 & 0.000476 & 0.000704 & 0.01000 & 0.001182 & 0.001289 & 0.001294 & 0.001302 \\
\hline
\end{tabular}

Table 2. Summary statistics for the regression models using tornado intensity values and Geary's $C$ values of all the image difference bands using a $21 \times 21$ window.

\begin{tabular}{lllllll}
\hline \multicolumn{7}{c}{ Geary' $C$ Bands $(21 \times 21)$} \\
\hline & Band 1 & Band 2 & Band 3 & Band 4 & Band 5 & Band 6 \\
\hline Correlation & 0.07 & 0.22 & 0.24 & 0.20 & 0.33 & 0.37 \\
Coef. of Det. & $0.50 \%$ & $4.72 \%$ & $5.66 \%$ & $3.95 \%$ & $11.19 \%$ & $13.53 \%$ \\
S.E. Estimate & 1.17 & 1.14 & 1.14 & 1.15 & 1.10 & 1.09 \\
Std. of X & 1282.56 & 1143.80 & 1220.08 & 1113.24 & 1268.54 & 1264.61 \\
Std. of Y & 1.17 & 1.17 & 1.17 & 1.17 & 1.17 & 1.17 \\
Intercept & 4.28 & 3.27 & 3.13 & 3.22 & 2.49 & 2.18 \\
Coefficient & 0.070658 & 0.000222 & 0.000228 & 0.000210 & 0.000308 & 0.000340 \\
\hline
\end{tabular}

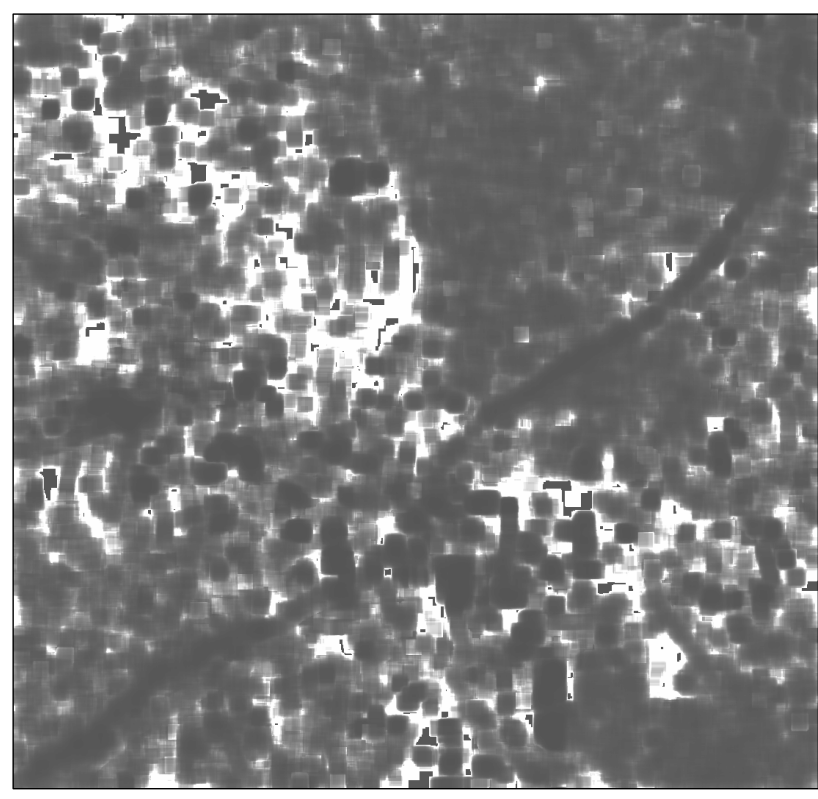

Fig. 9. Lacunarity differential box counting indices of image difference band 3 using $21 \times 21$ window. sides of the image (where $\mathrm{n}=$ window size), we performed a mirror extension of $(\mathrm{n}-1) / 2$ pixels around the image before computing spatial indices (Myint, 2006). This procedure applies to all selected geospatial approaches. We performed the regression and correlation analysis between geospatialtransformed values obtained in different window sizes and tornado intensity values. Figures $6,7,8$, and 9 show spatialtransformed images of the selected geospatial approaches (i.e., $G y, G i, L 1$, and $L 2$ ) using the band 3 difference image. A flow chart that demonstrates a step-by-step procedure to conduct this research study is presented in Fig. 10.

\section{Results and discussion}

We used the $G i$ approach to test different local window sizes (i.e., $5 \times 5,9 \times 9,13 \times 13,17 \times 17,21 \times 21,25 \times 25,29 \times 29$, $33 \times 33,37 \times 37)$ using the band 3 difference image to determine the optimal window. It is evident from Table 1 that the $21 \times 21$ window produced the highest correlation and coefficient determination, and the lowest standard error of estimate $(-0.53,27.78 \%, 0.99)$. The second highest correlation $(-0.51)$ was produced by the $25 \times 25$ window. Since larger window covers more land cover classes and non-damaged areas, the correlation can be expected to be low for a window that covers an extensively large area including non-damaged 
Table 3. Summary statistics for the regression models using tornado intensity values and Getis values of all the image difference bands using a $21 \times 21$ window.

\begin{tabular}{lllllll}
\hline \multicolumn{7}{c}{ Getis Index Bands $(21 \times 21)$} \\
\hline & Band 1 & Band 2 & Band 3 & Band 4 & Band 5 & Band 6 \\
\hline Correlation & -0.23 & -0.41 & -0.53 & 0.05 & -0.49 & -0.29 \\
Coef. of Det. & $5.43 \%$ & $17.08 \%$ & $27.78 \%$ & $0.23 \%$ & $24.12 \%$ & $8.59 \%$ \\
S.E. Estimate & 1.14 & 1.07 & 0.99 & 1.17 & 1.02 & 1.12 \\
Std. of X & 886.39 & 954.48 & 616.71 & 1095.85 & 605.42 & 407.39 \\
Std. of Y & 1.17 & 1.17 & 1.17 & 1.17 & 1.17 & 1.17 \\
Intercept & 5.35 & 5.77 & 6.53 & 4.52 & 6.34 & 6.03 \\
Coefficient & 0.000308 & 0.000506 & 0.001000 & 0.000510 & 0.000949 & 0.000841 \\
\hline
\end{tabular}

Table 4. Summary statistics for the regression models using tornado intensity values and lacunarity probability $(L 1)$ values of all the image difference bands using a $21 \times 21$ window.

\begin{tabular}{lllllll}
\hline \multicolumn{7}{c}{ L1 Bands (21×21) } \\
\hline & Band 1 & Band 2 & Band 3 & Band 4 & Band 5 & Band 6 \\
\hline Correlation & 0.26 & 0.22 & 0.40 & 0.03 & 0.32 & 0.46 \\
Coef. of Det. & $6.60 \%$ & $4.63 \%$ & $16.29 \%$ & $0.09 \%$ & $10.00 \%$ & $21.61 \%$ \\
S.E. Estimate & 1.13 & 1.14 & 1.07 & 1.17 & 1.11 & 1.04 \\
Std. of X & 64.68 & 1044.46 & 65.641 & 77.48 & 76.39 & 103.98 \\
Std. of Y & 1.17 & 1.17 & 1.17 & 1.17 & 1.17 & 1.17 \\
Intercept & 3.69 & 3.83 & 2.44 & 4.80 & 2.82 & 2.56 \\
Coefficient & 0.004647 & 0.003901 & 0.007171 & 0.000456 & 0.004842 & 0.005230 \\
\hline
\end{tabular}

areas. As anticipated, smaller window sizes (i.e., $5 \times 5,9 \times 9$ ) do not show any correlations and were found to be ineffective. This is probably due to the fact that smaller windows are not sufficiently large to cover tornado damaged areas completely, and geospatial algorithms may not be able to characterize spatial arrangements of damaged structures and debris including broken branches, stems, and leaves within a small window $(28.5 \mathrm{~m} \times 5=142.5 \mathrm{~m})$.

We therefore consider the $21 \times 21$ window to be the optimal window size to quantify tornado intensity values. Hence, we proceeded to perform the regression analysis between all the spatial-transformed images of $G y, G i, L 1$, and $L 2$ using all bands of difference images using the 21x21 window size. Summary statistics for the regression models using Fscale damage values and all the spatial-transformed images $G y, G i, L 1$, and $L 2$ using the $21 \times 21$ window size are presented in Tables 2, 3, 4, and 5 respectively.

With regard to the Geary's C-transformed images, band 6 of the Geary's C index gave the highest correlation coefficient and the lowest standard error of estimate $(0.37,1.09)$. Bands 1 and 4 were found to be ineffective since they show very low or no correlation with tornado damage categories $(0.07,0.20)$. Band 5 of Geary's $\mathrm{C}$ was found to be the second best band to identify tornado classification values. Band 3 had the highest correlation and the lowest standard error of estimate $(0.53,0.99)$ and hence, it was the most effective band of all the Getis transformed bands. Bands 2 and 4 of Getis indices were comparable to band 3 and found to be effective $(-0.41,0.49)$. There was no correlation found between band 4 and the NOAA F-scale damage assessment. Band 6 of the lacunarity probability approach had the highest correlation and the lowest standard error of estimate $(0.46,1.04)$. No correlation was evident between band 4 and NOAA tornado intensity values. Band 3 produced the second highest correlation and the second lowest standard error of estimate $(0.40,1.07)$. From Table 5 , it can be observed that band 3 of the lacunarity differential box counting approach produced the highest correlation and the lowest standard error of estimate $(-0.42,1.06)$. Band 4 of the lacunarity differential box counting approach showed no relation with tornado F-scale values. Band 5 of the same lacunarity approach gave the second highest correlation $(-0.32)$. In general, bands 3,5, and 6 of all the geospatial approaches were found to effectively quantify F-scale damage values. Conversely, Band 4 of all approaches showed the weakest relationship with F-scale damage intensities. This could have been due to the fact that band 4 (near infrared band) is sensitive to chlorophyll content in vegetation and consequently has little linkage to damaged areas and the complex spatial arrangements of man-made structures. 
Table 5. Summary statistics for the regression models using tornado intensity values and lacunarity differential box counting ( $L 2)$ values of all the image difference bands using a $21 \times 21$ window.

\begin{tabular}{lllllll}
\hline \multicolumn{7}{c}{ L2 Bands (21×21) } \\
\hline & Band 1 & Band 2 & Band 3 & Band 4 & Band 5 & Band 6 \\
\hline Correlation & -0.11 & -0.25 & -0.42 & -0.04 & -0.32 & -0.27 \\
Coef. of Det. & $1.27 \%$ & $6.23 \%$ & $17.69 \%$ & $0.19 \%$ & $10.43 \%$ & $7.03 \%$ \\
S.E. Estimate & 1.16 & 1.13 & 1.17 & 1.17 & 1.11 & 1.13 \\
Std. of X & 882.96 & 1044.46 & 466.63 & 2093.40 & 1256.93 & 259.58 \\
Std. of Y & 1.17 & 1.17 & 1.17 & 1.17 & 1.17 & 1.17 \\
Intercept & 4.75 & 4.82 & 5.07 & 4.64 & 4.81 & 4.99 \\
Coefficient & -0.000149 & -0.000280 & -0.001054 & -0.000024 & -0.000300 & -0.001150 \\
\hline
\end{tabular}

Table 6. Summary statistics of a multiple regression model using tornado intensity values and band 3 of all spatial-transformed images using a $21 \times 21$ window.

\begin{tabular}{lllrr}
\hline \multicolumn{2}{l}{ Multiple Regression Model 1 } & Independent & \\
& & Variables & Coefficient & t_test (2320) \\
\hline Apparent R & 0.5710 & Intercept & 4.954748 & 23.66 \\
Adjusted R & 0.5703 & Gi-Band3 & -0.000682 & -13.96 \\
Apparent R & $32.61 \%$ & Gy-Band3 & -0.000012 & -0.66 \\
Adjusted R & $32.52 \%$ & L1-Band3 & 0.003808 & 0.11 \\
F $(4,2320)$ & 280.65 & L2-Band3 & -0.000317 & -5.59 \\
\hline
\end{tabular}

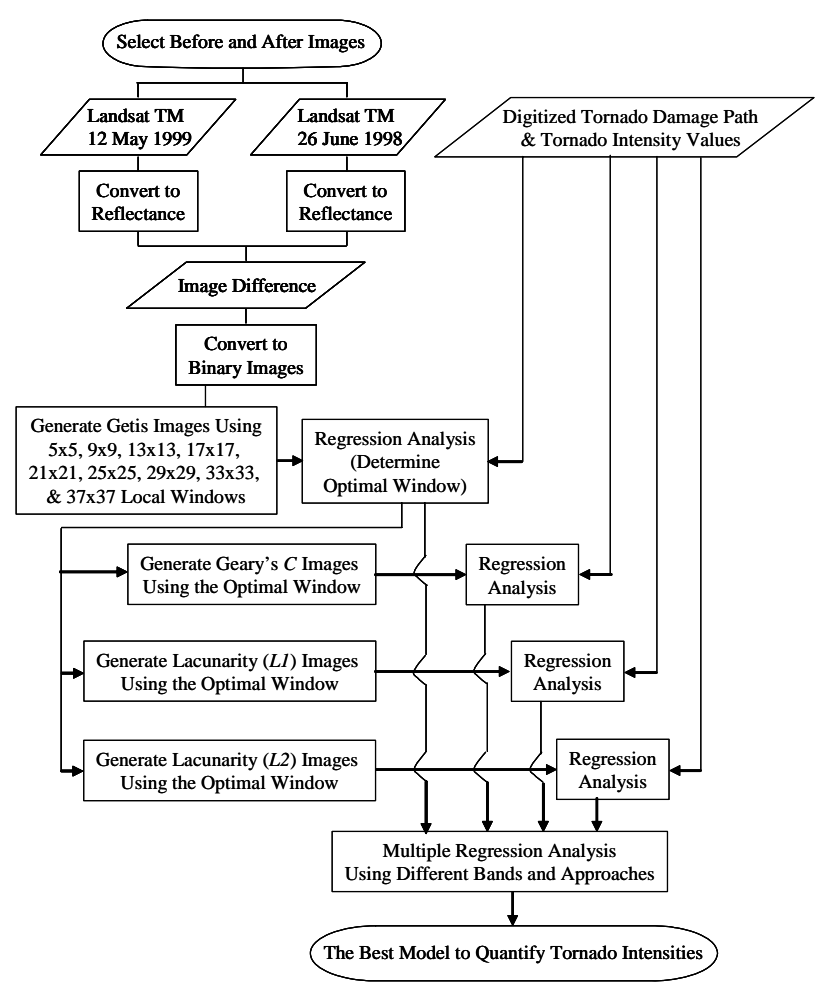

Fig. 10. Research Design.
Since band 3 of all spatial approaches was found to be the most effective band in relating to F-scale damage categories, we used band 3 of all spatial techniques to develop a multiple regression model to quantify F-scale damage categories. A summary statistics of the model is presented in Table 6. The multiple regression model is relatively more effective than the best single band linear regression model since the coefficient of determination given by the best single band model $(27.78 \%)$ is lower than that of the multiple regression model $(32.61 \%)$. We also attempted another multiple regression using bands 3,5 , and 6 of lacunarity probability $(L 1)$ indices and Getis indices. From Table 7, it can be observed that this multiple model is more powerful than the multiple model with band 3 of all spatial approaches since the apparent $R^{2}(40.69 \%)$ of this approach is higher than the first multiple model. We therefore suggest the multiple regression model incorporating bands 3,5 , and 7 of the $L 2$ and $G i$ approaches as the best model to quantify tornado F-scale damage values.

\section{Conclusions}

Our purpose has been to introduce a remote sensing approach to natural disaster damage assessment, and specifically in this case study, tornado damage assessment. Remote sensing of natural disaster damage offers exciting possibilities as a backup or, in some cases, an alternative to traditional 
Table 7. Summary statistics of a multiple regression model using tornado intensity values and bands 3,5 , and 6 of lacunarity probability and Getis indices using a $21 \times 21$ window.

\begin{tabular}{|c|c|c|c|c|}
\hline \multirow{2}{*}{\multicolumn{2}{|c|}{ Multiple Regression Model 2}} & \multicolumn{3}{|c|}{ Independent } \\
\hline & & Variables & Coefficient & t_test (2318) \\
\hline Apparent $\mathrm{R}$ & 0.6379 & Intercept & 4.376841 & 24.57 \\
\hline Adjusted R & 0.6369 & L1-Band3 & 0.002915 & 8.64 \\
\hline Apparent $\mathrm{R}^{2}$ & $40.69 \%$ & $L 1$-Band5 & -0.002078 & -6.31 \\
\hline Adjusted $\mathrm{R}^{2}$ & $40.56 \%$ & L1-Band6 & 0.003352 & 13.84 \\
\hline \multirow[t]{3}{*}{$\mathrm{F}(4,2320)$} & 265.02 & Gi-Band3 & -0.000570 & -9.76 \\
\hline & & Gi-Band5 & -0.000559 & -7.98 \\
\hline & & Gi-Band6 & 0.000544 & 6.60 \\
\hline
\end{tabular}

on-site surveys because of satellite imagery's global ubiquity, its ability for repeated independent analysis, and, as we demonstrated here, its ability to verify on-site damage assessment. Using the massive tornado event of 3 May 1999 as a case example, we digitized the tornado damage path and co-registered the damage path using pre- and post-Landsat Thematic Mapper image data of the event at $28.5 \mathrm{~m}$ spatial resolution with seven channels ranging from blue to thermal infrared portion of the spectrum was used to perform a damage assessment. Our approach examined the correlation between F-scale measures and homogeneity of spectral reflectance. We employed several geospatial approaches, specifically employed the Getis index, Geary's $C$, and two lacunarity approaches, to categorize the imagery to groundsurvey F-scale tornado destruction values.

We have determined that there are strong relationships between spatial indices computed within a local window and F-scale damage categories identified through the ground survey. Consequently, linear regression models, even incorporating just a single band, prove to be effective in establishing F-scale damage categories using satellite imagery. The optimal window size identified in the study was $21 \times 21$. A window size smaller than $9 \times 9(256.5 \mathrm{~m})$ was not useful for classifying to tornado F-scale damage values. As expected earlier, multiple regression models outperformed the single band regression models.

It should be noted that F-scale damage class estimates were assigned by the on-site surveyors, and we considered these values as reference values or ground-truth values. In other words, our study is based on the assumption that the ground survey was completely accurate. On the other hand, these values were assigned to the digitized map at some approximate locations along the tornado damage track in comparison to the tornado intensity map prepared by the National Oceanic and Atmospheric Administration (NOAA) National Weather Service. Although potential error exists in comparing on-site point data ground estimates into a digital dataset, we believe that both single-band models and multiple regression models developed in this study are effective. Since we used apparent surface reflectance of the Landsat data, the regression models developed in the study can be employed to identify F-scale damage categories for any future tornado event using the same satellite data as a quick assessment before conducting the ground survey, a guide to characterize damage intensities more efficiently on the ground, or a supplementary approach for identifying tornado categories more accurately. A similar approach with the use of any simple statistical filter or advanced geospatial approach can be used effectively to assess damage intensities for any other tornado event and any other natural disaster (e.g., damages due to a tsunami event, a cyclone, or a hurricane).

Although satellite imagery analysis as a form of damage assessment offers exciting new possibilities, particularly for remote locations where on-site surveying can be problematic, some potential problems do exist. For example, satellite imagery is not yet available for all locations at all times (because of coverage limitations) and, occasionally, costs or acquisition rights for imagery can be prohibitive depending on company, agency and/or government associated with that imagery. In addition, some uncertainties and limitations may exist in the digital form of verification damage values used to compare our results. Those limitations notwithstanding, this study demonstrates that geospatial techniques with the use of difference images can be employed effectively to classify natural disaster damage categories, in this case that associated with tornadoes.

Acknowledgement. This research has been supported by the National Science Foundation (grant \# BCS-0649413).

Edited by: K. Chang

Reviewed by: two anonymous referees 


\section{References}

Arciniegas, G. A., Biker, W., Kerle, N., and Tolpekin, V. A.: Coherence- and amplitude-based analysis of seismogenic damge in Bam, Iran, using ASAR data, IEEE Transactions on Geoscience and Remote Sensing, 45, 1571-1581, 2007.

Bach, J., Pascual, R., Rigo, T., Pineda, N., López, J. M., Arús, J. and Gayà, M.: An observation study of the 7 September 2005 Barcelona tornado outbreak, Nat. Hazards Earth Syst. Sci., 7, 129-139, 2007, http://www.nat-hazards-earth-syst-sci.net/7/129/2007/.

Barnes, C. F., Fritz, H., and Jeseon, Y.: Hurricane disaster assessments with image-driven data mining in high-resolution satellite imagery, IEEE Transactions on Geoscience and Remote Sensing, 45, 1631-1640, 2007

Bentley, M. L., Mote, T. L., and Thebpanya, P: Using Landsat to identify thunderstorm damage in agricultural regions, Bull. Amer. Met. Soc., 83, 363-376, 2002

Bovolo, F. and Bruzzone, L.: A split-based approach to unsupervised change detection in large-size multitemporal images: Application to tsunami-damage assessment, IEEE Transactions on Geoscience and Remote Sensing, 45, 1658-1670, 2007

Bunting, W. F. and Smith, B. E.: A guide for conducting damage surveys, NOAA Tech. Memo, NWS SR-146, 44 pp. [NTIS Accession No. PB93-148427INZ], 1990.

Allain, C. and Cloitre, M.: Characterizing the lacunarity of random and deterministic fractal sets, Physics Review, A, 44, 552-3558, 1991.

Cerveny, R. S., Lawrimore, J., Edwards, R., and Landsea, C.: Extreme Weather Records: Compilation, Adjudication and Publication, Bull. Amer. Meteor. Soc., 88, 853-860, 2007.

Chaabane, F., Avallone, A., Tupin, F., Briole, P., and Maitre, H: A multitemporal method for correction of tropospheric effects in differential SAR Interferometry: Application to the Gulf of Corinth earthquake, IEEE Transactions on Geoscience and Remote Sensing, 45, 1605-1615, 2007.

Chavez, P. S.: Image-Based Atmospheric Corrections - Revisited and Improved, Photogrammetric Engineering and Remote Sensing, 62, 1025-1036, 1996.

Dong, P.: Lacunarity for spatial heterogeneity measurement in GIS, Geographic Information Sciences, 6(1), 20-26, 2000

Doswell, III, C. A.: Societal impacts of severe thunderstorms and tornadoes: lessons learned and implications for Europe, Atmos. Res., 67-8, 135-152, 2003.

Doswell III, C. A. and Burgess, D. W.: On some issues of United States tornado climatology, Mon. Wea. Rev., 116, 495-501, 1988.

Dyer, R. C.: Remote sensing identification of tornado tracks in Argentina, Brazil and Paraguay, Photogrammetric Engineering and Remote Sensing, 54, 1429-1435, 1988.

Edwards, R., Corfidi, S. F., Thompson, R. L., Evans, J. S., Craven, J. P., Racy, J. P., McCarthy, D. W. and Vescio, M. D.: Storm Prediction Center forecasting issues related to the 3 May 1999 tornado outbreak, Weather and Forecasting, 17, 544-558, 2002.

Fujita, T. T.: Proposed characterization of tornadoes and hurricanes by area and intensity, SMRP Research Rep. 91, University of Chicago, Chicago, IL., 15 pp., 1971.

Fujita, T. T.: Experimental classification of tornadoes in FPP scale, SMRP Research Rep. 98, University of Chicago, Chicago IL., 15 pp., 1973.
Fujita, T. T.: Tornadoes and downbursts in the context of generalized planetary scales, J. Atmos. Sci., 38, 1511-1534, 1981.

Fujita, T. T. and Smith, B. E.: Aerial survey and photography of tornado and microburst damage. The Tornado: Its Structure, Prediction and Hazards, Geophys. Monogr. No. 79, Amer. Geophys. Union, 479-493, 1993.

Getis, A. and Ord, J. K.: The analysis of spatial association by use of distance statistics, Geographical Analysis, 24(3), 1269-1277, 1992.

Jedlovec, G. J., Nair, U. and Haines, S. L.: Detection of storm damage tracks with EOS data, Weather and Forecasting, 21, 249-267, 2006.

Marshall, T. P.: Tornado damage survey at Moore, Oklahoma, Weather and Forecasting, 17, 582-598, 2002.

McDonald, J. and Mehta K.C.: A Recommendation for an Enhanced Fujita Scale (EF-Scale), Lubbock, Texas: Wind Science and Engineering Research Center, http://www.wind.ttu.edu/ EFScale.pdf, 2006.

Myint, S. W. and Lam, N. S. N.: Examining Lacunarity A.pproaches in Comparison with Fractal and Spatial Autocorrelation Techniques for Urban Mapping, Photogrammetric Engineering and Remote Sensing, 71, 927-937, 2005.

Myint, S. W., Mesev, V., and Lam, N. S. N.: Texture Analysis and Classification Through A Modified Lacunarity Analysis Based on Differential Box Counting Method, Geographical Analysis, 38(2006), 371-390, 2006.

Myint, S. W. and Wang, L.: Multi-criteria Decision Approach for Land Use Land Cover Change Using Markov Chain Analysis and Cellular Automata Approach, Canadian Journal of Remote Sensing, 32, 390-404, 2006.

Myint, S. W., Wentz, E., and Purkis, S.: Employing spatial metrics in urban land use/land cover mapping: comparing the Getis and Geary indices, Photogrammetric Engineering and Remote Sensing, 73(21), 1403-1415, 2007.

Oosterom, P. J. M., Zlatanova S. and Fendel, E. M.: International Symposium on Geo-Information for Disaster Management, Springer-Verlag, Berlin, 1434, 2005.

Ord, J. and Getis, A.: Local spatial autocorrelation statistics: Distributional issues and an application, Geographical Analysis, 27, 286-306, 1995.

Reese, S., Cousins, W. J., Power, W. L., Palmer, N. G., Tejakusuma, I. G., and Nugrahadi, S.: Tsunami vulnerability of buildings and people in South Java - field observations after the July 2006 Java tsunami, Nat. Haz. Earth Sys. Sci., 7, 573-589, 2007.

Inoue S., Wijeyewickrema, A. C., Matsumoto, H., Miura, H., Gunaratna, P., Madurapperuma, M., and Sekiguchi, T.: Field survey of tsunami effects in Sri Lanka due to the Sumatra-Andaman Earthquake of 26 December 2004, Pure Appl. Geophys, 164, 395-411, 2007.

Sertel, E., Kaya, S. and Curran, P. J.: Use of semivariograms to identify earthquake damage in an urban area, IEEE Transactions on Geoscience and Remote Sensing, 45, 1590-1594, 2007.

Smith III, T.J. and Robblee, M. B.: Mangroves, hurricanes and lightning strikes, Bioscience, 44, 256-263, 1994.

Speheger D. A., Doswell III, C. A., and Stumpt, G. J.: The tornadoes of 3 May 1999: Event verification in central Oklahoma and related issues, Wea. Forecasting, 17, 362-381, 2002.

Simpson, R. H.: The hurricane disaster potential scale, Weatherwise, $27,169,186,1974$ 
Yuan, D., Elvidge, C. D. and Lunetta, R.: Survey of multispectral methods for land cover change analysis. In Remote Sensing Change Detection, Environmental Monitoring Methods and Applications, Edited by R. Lunetta, and C.D. Elvidge, (Ann Arbor press, Michigan), 1-19, 1998.

Yuan, M., Dickens-Micozzi, M., and Magsig, M. A.: Analysis of tornado damage tracks from the 3 May tornado outbreak using multispectral satellite imagery, Weather and Forecasting, 17(3), 382-398, 2002.
Zlatanova, S. and Li, J.: Geospatial Information Technology for Emergency Response (ISPRS book series, number 6), Taylor and Frances Group, London, 381, 2008. 